\title{
An Open-Access Atom Probe Tomography Mass Spectrum Database
}

\author{
David R. Diercks ${ }^{1}$, Brian P. Gorman ${ }^{1}$ and Stephan S.A. Gerstl ${ }^{2}$ \\ 1. Colorado School of Mines, Department of Metallurgical and Materials Engineering, Golden, CO USA \\ 2. ETH Zurich, Scientific Center for Optical \& Electron Microscopy (ScopeM), Zurich, Switzerland
}

Atom probe tomography as a reliable and accessible materials characterization technique has greatly expanded within the past decade. Along with that expansion has come the published analyses of a greater variety of materials [1]. While these have demonstrated a number of significant discoveries, many of the details and pragmatic aspects get overlooked. Among those details are the time-of-flight (TOF) mass spectra and associated collection parameters. Often, it is the knowledge of these facets that are critical for expanding the technique to additional materials or new applications. In order to facilitate the broad dissemination of such useful information, an open-access, online database has been created http://atomprobe.mines.edu/mass-spectrum-database.html.

The database contains representative atom probe mass spectra for a number of materials and will grow as users contribute more of their results. It maintains with a mass spectrum, a record of several important instrument parameters including the instrument type, pulsing mode, base temperature, pulse frequency, laser energy, detection rate, voltage range, and flight path length. Also, a number of material and specimen specific variables are documented, including phase, composition, specimen preparation $\operatorname{method}(\mathrm{s})$, and specimen images. Additionally, there are fields for a number of metrics regarding the mass spectra including measured number of multiples, measured background, and mass resolving power. Attachment files such as images, two column text files, and range files can be included through a drag and drop feature. Table 1 presents an overview of the entries and entry types included in the database.

As mentioned above, the database is open-access and is available for any researcher to add relevant TOF mass spectra. While this is envisioned as a collection of spectra from the atom probe community as a whole, it will be moderated to ensure that the entries are appropriately complete, representative, and not overly redundant. That is, the spectra should be sufficiently distinct from each other to offer information that is useful to other atom probe users. It will not be merely a repository of every single APT analysis ever collected.

There are several anticipated applications of this database. First, it will be quite useful to see if anyone has run a particular type of sample before without having to comb through the literature. Since the database is searchable and filterable by a number of parameters, this can be easily ascertained while also directing the user to related publications. Second, it allows users to see which data collection conditions have been used, and possibly optimized, for particular types of materials. This may lead to more quickly achieving useful results on new projects without having to spend as much time and funding experimenting to achieve the best conditions for high-confidence results. Third, it can be a repository for useful details that often don't make it into publications such as voltage range and percentage of multiple hits observed. Fourth, it can provide an indication beforehand of what to expect in the mass spectrum for a material and what might be achievable. For example, a user may be able to assess the likely detectability of trace species based on the background values others have measured or identify or corroborate likely mass peak interferences. Fifth, a central and online repository with the ability to filter 
by various parameters (with optional CSV export enabling further analysis) may make it easier to draw connections and elucidate trends that would not otherwise be apparent [2].

\section{References:}

[1] A. Devaraj et al, International Materials Reviews (2017) p. 1.

[2] The authors thank Hugues G. Francois-Saint-Cyr, Ann N. Chiaramonti Debay, and Paul Blanchard for providing helpful feedback.

Table 1. Overview of the data and data types contained in the Open-Access Atom Probe Tomography Mass Spectrum Database.

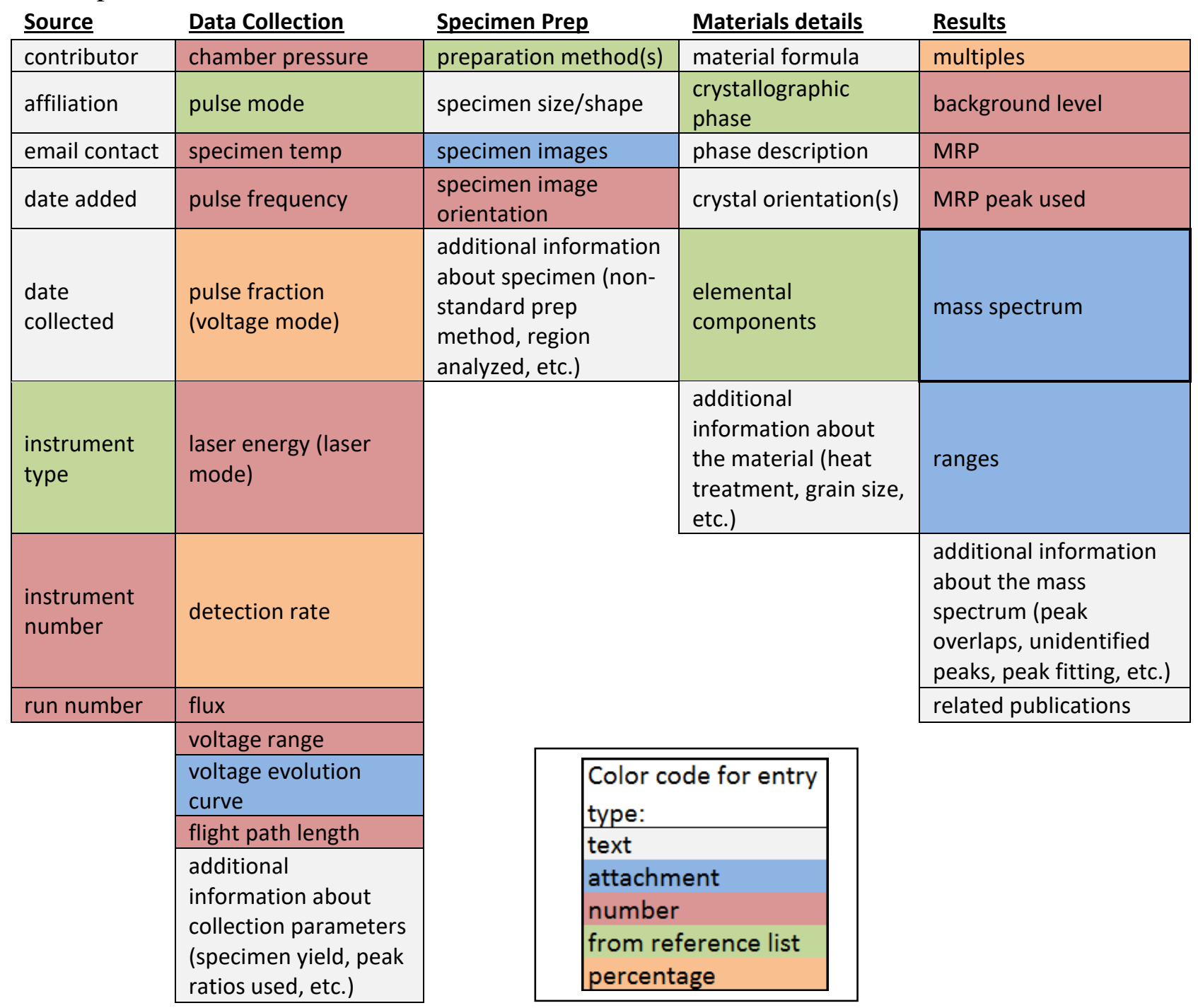

